\title{
Sars-CoV-2 Infection in a Neutropenic Pediatric Patient with Leukemia: Addressing the need for universal guidelines for treatment of Sars-CoV-2 positive, immunocompromised patients
}

\author{
Anastasia Schied $^{1}$, Erin Trovillion ${ }^{1}$, and Amaran Moodley ${ }^{1}$ \\ ${ }^{1}$ University of California San Diego
}

June 12, 2020

\begin{abstract}
In December 2019, a novel coronavirus (Sars-CoV-2 ) emerged from Wuhan, Hubei province, China, and has spread worldwide, with more than 4 million confirmed cases[1,2,3]. Available literature on the clinical characteristics and natural history of pediatric patients infected with Sars-CoV-2 is limited and less has been established regarding guidelines for the treatment of immunocompromised pediatric patients. This is a case report on the clinical course and management of a 6 year old female with acute lymphoblastic lymphoma with Sars-CoV-2. The goal is to highlight the need for management guidelines in the care of pediatric cancer patients with Sars-CoV-2.
\end{abstract}

\section{INTRODUCTION:}

\section{Patient Presentation:}

The patient is a 6 year old female with B-lymphoblastic lymphoma who is in the maintenance phase of treatment, as per Children's Oncology Group (COG) study AALL1131 High Risk Arm. Her family reported a fever of 101.8 Fahrenheit so she was directed to the emergency room for evaluation of fever in an immunocompromised patient with a central line. She was found to have cytopenias secondary to chemotherapy, with a white blood cell count (WBC) of $1.4 \mathrm{TH} / \mathrm{uL}$, hemoglobin of $7.7 \mathrm{~g} / \mathrm{dL}$, platelet of $229 \mathrm{TH} / \mathrm{uL}$, and absolute neutrophil count (ANC) of 140/uL. Given the severe neutropenia she was started on empiric broad spectrum antibiotics and was admitted per hospital standard of care.

History was significant for multiple symptomatic family household members who had tested positive for Sars$\mathrm{CoV}-2$. The index cases were her grandmother and grandfather who had exposure to Sars-CoV-2 positive patients at an adult hospice where they were employed. The patient had a history of 1 day of cough, but was asymptomatic on admission and remained afebrile throughout her hospitalization. She denied symptoms of cough, shortness of breath, fatigue, abdominal pain, or diarrhea during her 10 day admission.

\section{Diagnostic/Therapeutic Focus and Assessment:}

While in the Emergency Department, the patient underwent diagnostic testing that included a nasopharyngeal (NP) swab for detection of SARS-CoV-2 and other respiratory pathogens by polymerase chain reaction (PCR), blood culture and urine culture. She received a normal saline bolus, was started on intravenous (IV) cefepime, and admitted to a single-patient, negative pressure room in the Special Infectious Disease Unit (SIDU). Infectious disease testing was positive for SARS-CoV-2 by NP PCR, negative for other respiratory virus co-infections, and negative blood and urine cultures.

The patient was transfused for a hemoglobin of 7.7, particularly in the context of SARS-CoV-2 infection. Infectious Disease specialists and Infection Control were consulted and recommended a baseline chest X- 
ray $(\mathrm{CXR})$ and inflammatory markers which were monitored as her clinical condition evolved during her hospitalization. Table 1 highlights important lab values. In particular, D-Dimer, lactate dehydrogenase (LDH), and interleukin 6 (IL-6) were not elevated, and trended down during admission. Her ferritin was slightly elevated at 1,098, and down trended to 983 on repeat testing. Her CXR was normal, and as her clinical status remained stable without shortness of breath, cough, hypoxia, or increased work of breathing, imaging was not repeated prior to discharge. Her oral chemotherapy was held while neutropenic as per COG AALL1131 protocol guidelines and while actively infected with the virus.

\section{Follow up and Outcome:}

The patient was discharged home after 10 days of hospitalization. Her blood cultures were negative, she was asymptomatic and remained afebrile after antibiotics were discontinued, and her ANC was 162/uL at discharge. She had repeat Sars-CoV-2 PCR testing of a naso-pharyngeal swab 3 weeks after her diagnosis and it was negative. Her cytopenias had resolved and her oncology team proceeded with Vincristine therapy, prednisone pulse dosing, and restarted her oral mercaptopurine and methotrexate. Serum Sars-CoV-2 IgG testing at 6 weeks after diagnosis was positive; demonstrating that she did mount an antibody response while immunocompromised. From the patient and patient's family perspective, the extended hospital stay was appreciated as necessary to keep the patient safe. They were receptive to holding chemotherapy while she was actively infected, and agreed with conservative management in the context of possible increased risk for severe course in immunocompromised patients.

\section{DISCUSSION:}

This case report provides an example of an immunocompromised pediatric patient who had a favorable course and outcome after infection with Sars-CoV-2. She was treated with supportive care only, and did not receive any experimental antiviral therapies or intravenous immunoglobulin. In the pediatric population overall, rate of hospitalization is lower (5.7\% compared to $20 \%$ in adults), and most recover within 1-2 weeks of symptom onset. ${ }^{[4,5]}$ It is still unclear why younger people are not as severely affected by the virus, but theories attributing differences in immune system function prompt additional questions regarding oncology patients. ${ }^{6}$ The literature available is limited, but our patient has followed a similarly mild course to 5 case reports from a childhood cancer center in Lombardy Italy. ${ }^{7}$ Some of those patients were managed at home, rather than admitted to the hospital. We do not yet have specific guidelines for admission or discharge criteria in the United States for pediatric oncology patients who contract the virus. Our Infectious Diseases team recommended baseline CXR, as well as trending certain inflammatory markers based on findings in the adult literature, IL-6 in particular ${ }^{8}$. Other possible guidelines suggested in the World Journal of Pediatrics recommend the following criteria be met prior to discharge: Afebrile for 3 days, absence of respiratory distress, and two negative Sars-CoV-2 tests.

Although a small number of case reports have demonstrated mild clinical courses for pediatric oncology patients, there is evidence that an immunocompromised state is associated with higher rates of Lower Respiratory Tract Disease (LRTD). ${ }^{9}$ In a study performed by Ogimi $\mathrm{C}$ et al. in Seattle, Washington, the characteristics of coronavirus infection in immunocompromised as well as immunocompetent pediatric patients were investigated, specifically the prevalence of LRTD, which is an indicator of more severe disease and worse prognosis in Sars-CoV-2 cases. The difference in prevalence was not statistically significant, but in a multivariable model, there was an increased likelihood of severe LRTD associated with an immunocompromised state (adjusted odds ratio, 2.5 [95\% confidence interval, 1.2-4.9]; P =.01). ${ }^{9}$ Until we have gathered more robust data on the clinical course of immunocompromised patients, it remains prudent to observe and monitor these patients conservatively if they contract Sars-CoV-2.

Current recommendations for pediatric oncology patients are being published by various specific oncology organizations. ${ }^{[7,10,11]}$ A general theme is to reduce intense chemotherapy and prolong intervals between cycles in patients with stable disease. For asymptomatic oncology patients, particularly sarcoma patients, surgery should not be delayed despite the aerosolizing procedures required for operative management. In immunocompromised patients who are found positive for Sars-CoV-2, the French Sarcoma Group recom- 
mends delaying treatment for at least 15 days after symptom onset, similar to how we proceeded in this case report. ${ }^{11}$

While caring for this patient, we identified multiple areas where unified guidelines for patient care would be beneficial.

1. Admission criteria, routine lab monitoring and imaging recommended, possible treatment options/protocols, and discharge criteria.

2. Specific follow up testing/monitoring in immunocompromised patients.

3. Guidelines for adjustments in chemotherapy, radiation, and surgical interventions for pediatric oncology patients.

4. It will be important to understand which patients may benefit from various experimental therapies. ${ }^{12}$

We have a need as providers to further investigate how immunocompromised children are responding differently to the virus, and the community of patient families needs clear recommendations. For example, additional precautions these families should take as the country begins reopening, information on how the clinical course may differ in immunocompromised children, and how treatment protocols may be adjusted during this pandemic. There has been some epidemiologic investigation into the perceived risk, and anxiety regarding the pandemic in pediatric oncology patients. In a survey of young cancer patients in Milan, a large proportion of them felt "personally at risk of severe complications". The SIOP, St. Jude, the American Society of Hematology, and others are actively developing a new platform for sharing resources and experiences in the evolving time of this global pandemic ${ }^{[5,13]}$. It will be important to continue sharing case information and conducting additional research in the coming years.

CONFLICT OF INTEREST STATEMENT: Authors do not have any conflicts of interest.

ACKNOWLEDGEMENTS: Consent was provided by the patient and her family to publish information regarding her medical condition and treatment.

REFERENCES:

1. Wang C, Horby PW, Hayden FG, Gao GF. A novel coronavirus outbreak of global health concern. Lancet 2020;395;470-473.

2. Xu Y, Li X, Zhu B, Liang H, Fang C, Gong Y, Guo Q, Sun X, Zhao D, Shen J, Zhang H, Liu H, Xia H, Tang J, Zhang K, and Gong S. Characteristics of pediatric SARS-CoV-2 infection and potential evidence for persistent fecal viral shedding. Nature Medicine, 26(4); 502-505.

3. Johns Hopkins Coronavirus Resource Center. 2020. COVID-19 Map . [online] Available at: <https://coronavirus.jhu.edu/map.html> [Accessed 11 May 2020].

4. Bialek S, Gierke R, Hughes M, McNamara L, Pilishvili T, Skoff T. Coronavirus Disease 2019 in Children - United States, February 12-April 2, 2020. MMWR. Morbidity and Mortality Weekly Report, $69(14) ; 422-426$.

5. Shen K, Yang Y, Wang T et al. Diagnosis, treatment, and prevention of 2019 novel coronavirus infection in children: experts' consensus statement. World J Pediatr (2020).

6. Mourey L, Falandry C, de Decker L, Boulahssass R, Carola E, Bengrine Lefevre L, Cudennec T, Brain E, Paillaud E, Soubeyran P. Taking care of older patients with cancer in the context of COVID-19 pandemic. The Lancet Oncology 2020;21(5), 236.

7. Bouffet E, Challinor J, Sullivan M, Biondi A, Rodriguez-Galindo C, Pritchard-Jones K. Early advice on managing children with cancer during the COVID-19 pandemic and a call for sharing experiences. Pediatric Blood \& Cancer 2020.

8. Aziz M, Fatima R, Assaly R. Elevated Interleukin-6 and Severe COVID-19: A Meta-Analysis. Journal of Medical Virology 2020.

9. Ogimi C, Englund JA, Bradford MC, Qin X, Boeckh M, Waghmare A. Characteristics and outcomes of coronavirus infection in children: the role of viral factors and an immunocompromised state. J Pediatric Infect Dis Soc 2019;8(1):21-28. 
10. Yang C, Li C, Wang S. Clinical strategies for treating pediatric cancer during the outbreak of 2019 novel coronavirus infection. Pediatric Blood \& Cancer 2020;67(5).

11. Penel N, Bonvalot S, Minard V, Orbach D, Gouin F, Corradini N, Brahmi M, Marec-Bérard P, Briand S, Gaspar N, Llacer C, Carrère S, Dufresne A, Le Cesne A, Blay J. French Sarcoma Group proposals for management of sarcoma patients during the COVID-19 outbreak. Annals of Oncology 2020.

12. Chiotos K, Hayes M, Kimberlin DW, Jones SB, James SH, Pinninti SG, Yarbrough A, Abzug MJ, MacBrayne CE, Soma VL, Dulek DE, Vora SB, Waghmare A, Wolf J, Olivero R, Grapentine S, Wattier RL, Bio L, Cross SJ, Dillman NO, Downes KJ, Timberlake K, Young J, Orscheln RC, Tamma PD, Schwenk HT, Zachariah P, Aldrich M, Goldman DL, Groves HE, Lamb GS, Tribble AC, Hersh AL, Thorell EA, Denison MR, Ratner AJ, Newland JG, Nakamura MM. Multicenter Initial Guidance on Use of Antivirals for Children With Coronavirus Disease 2019/Severe Acute Respiratory Syndrome Coronavirus 2. Journal of the Pediatric Infectious Diseases Society 2020.

13. Casanova M, Pagani Bagliacca E, Silva M, Patriarca C, Veneroni L, Clerici C, Spreafico F, Luksch R, Terenziani M, Meazza C, Podda M, Biassoni V, Schiavello E, Chiaravalli S, Puma N, Bergamaschi L, Gattuso G, Sironi G, Massimino M, Ferrari A.. How young patients with cancer perceive the COVID-19 (coronavirus) epidemic in Milan, Italy: Is there room for other fears? Pediatric Blood \& Cancer 2020.

14. Cruz A, Zeichner S. COVID-19 in Children: Initial Characterization of the Pediatric Disease. Pediatrics 2020.

15. Pathak E, Salemi J, Sobers N, Menard J, Hambleton I. COVID-19 in Children in the United States. Journal of Public Health Management and Practice 2020;1.

16. Balasubramanian S, Rao NM, Goenka A, Roderick M, Ramanan AV. Coronavirus Disease (COVID-19) in Children - What We Know So Far and What We Do Not? Indian Pediatr 2020.

17. Wang C, Ng C, Brook R. Response to COVID-19 in Taiwan. JAMA 2020;323(14);1341.

TABLE 1 Summary of Inflammatory Markers

\begin{tabular}{llll}
\hline & Day 1 & Day 3 & Day 5 \\
\hline D-Dimer (Ref: $<250 \mathrm{ng} / \mathrm{mL})$ & - & $<100$ & $<100$ \\
Lactate dehydrogenase (Ref: $470-900 \mathrm{u} / \mathrm{L})$ & 601 & - & 560 \\
Ferritin (Ref: 6-70 ng/mL) & 1,098 & - & 983 \\
Interleukin-6 (Ref: $<5.00 \mathrm{pg} / \mathrm{mL})$ & - & 2.77 & 1.81 \\
\hline
\end{tabular}

\section{FIGURE LEGEND:}

Figure 1 Chest X-ray with "No acute cardiopulmonary abnormality." obtained on Day 3 of hospitalization. 
Figure 1 Chest X-ray with "No acute cardiopulmonary abnormality." obtained on Day 3 of hospitalization

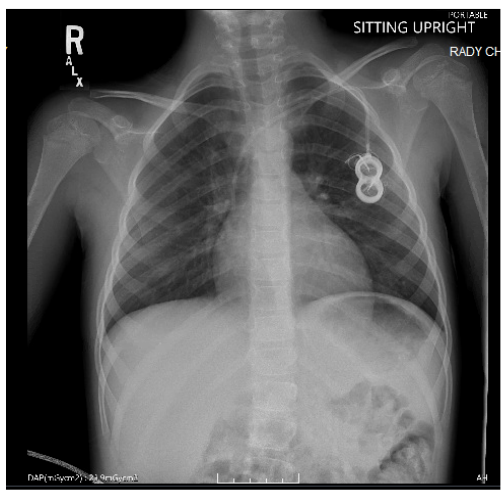

